\title{
Effect of Substrate on Structural Phase Transition in a Conducting Polymer during lon Injection and Water Intake: A View from a Computational Microscope
}

Sarbani Ghosh and Igor Zozoulenko*

Cite This: ACS Appl. Electron. Mater. 2020, 2, 4034-4041

Read Online

ABSTRACT: Conducting polymers operating in aqueous electrolyte represent mixed electron-ion conductors, where the ion injection and water intake can lead to structural and morphological changes that can strongly affect the material morphology and device performance. In the present paper, using molecular dynamics simulations, we provide an atomistic understanding of the structural phase transitions during electrochemical oxidation and ion injection in a conjugated polymer with glycolated side chains recently reported by Bischak et al. [J. Am. Chem. Soc., 2020, $142,7434]$, where the polymer switched between two structurally distinct phases corresponding to different oxidation levels. To outline the structural changes, we calculated the polymer film

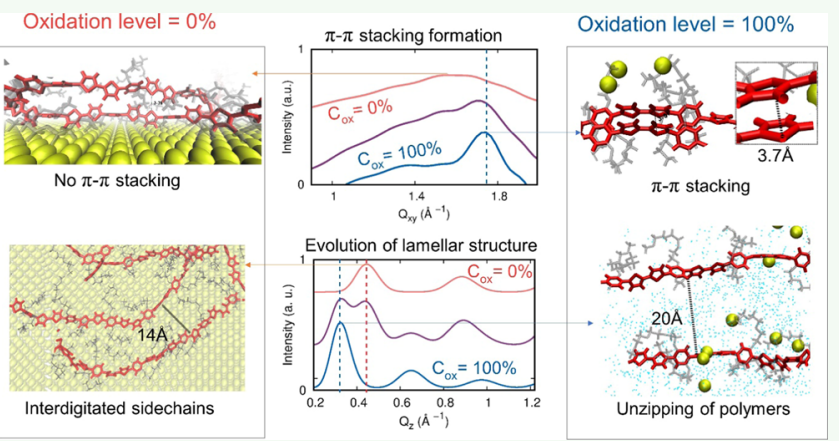
morphology and X-ray diffraction patterns at different oxidation levels. We demonstrated that the observed phase transition arises due to interplay between several factors, including the effect of the substrate leading to the preferential edge-on arrangement of the chains and formation of lamellas; unzipping of the interdigitated polymer chains during oxidation and ion intake; and changes in the morphology when $\pi-\pi$ stacking is absent at low oxidation level and forms at the high oxidation level facilitating the electron mobility and enabling the oxidation of the polymer film. Our calculations quantitatively reproduce the experimental data, which outlines the predictive power of the molecular modeling of the polymer systems that can be utilized for the design of materials and devices with improved performance.

KEYWORDS: conducting polymers, mixed electron-ion conductors, molecular dynamics simulations, computational microscopy, cyclic voltammetry, water intake

\section{INTRODUCTION}

Conducting polymers operating in an aqueous electrolyte represent mixed electron-ion conductors, and therefore they are widely utilized in a variety of devices where the coupling between the electronic and ionic motion is the prerequisite for the device functionality. ${ }^{1}$ Examples of such devices include supercapacitors, ${ }^{2}$ electrochromic displays, ${ }^{3}$ and batteries. ${ }^{4}$ Devices utilizing a mixed electron-ion conduction such as organic electrochemical transistors (OECT), ${ }^{5}$ neural probes, ${ }^{6}$ and ion pumps ${ }^{7}$ are the cornerstone of the organic bioelectronics as they provide the interface between the biological systems (operating with ions) and conventional electronics relying on electron signals.

Cyclic voltammetry is often used for the characterization of polymer-based mixed conductors. During the oxidation cycle, hydrated ions enter the polymer films from the electrolyte pushing the electrons residing at conjugated polymer backbones into the electrode. During the reduction cycle, the applied voltage forces the electrons from the electrode back into the polymer and the ions back into the electrolyte. ${ }^{8}$ During recent years, the ion injection and water or humidity intake in conducting polymers and hydrogels have been a subject of intense research. ${ }^{9-20}$ The water and ion intake often lead to structural and morphological changes that can strongly affect the material morphology and device performance. For example, careful engineering of the side chains of conjugated polymers resulted in improved control of ion injection and water intake, leading to boosting the stability and performance of OECTs. ${ }^{17,21}$ Ion injection led to a significant volume expansion ranging from 1000 to $10000 \%^{13}$ and to reversible structural phase transitions during cyclic voltammetry. ${ }^{19}$ While a significant amount of empirical knowledge is gained from the accumulated experimental results, in many cases, the underlying physical and electrochemical processes affecting and

Received: September 22, 2020

Accepted: December 2, 2020

Published: December 11, 2020 
changing the structural, morphological, and transport properties of conducting polymers are not understood theoretically. Such a detailed theoretical understanding is crucial, because without it, further progress in material improvement and device optimization is difficult.

Computational simulation and modeling providing theoretical understanding of material properties have already become a powerful tool for not only supporting but also, in many cases, guiding research in many areas of material science. The term "computationally microscopy" has been recently coined referring to simulation of structural properties on the time and length scales that are not accessible by conventional experimental techniques. ${ }^{22}$ The essential theoretical insight not available before has recently been gained from the molecular dynamics simulations of conducting polymers, e.g., for prediction of the morphology of the active layer of bulk heterojunction organic solar cells, ${ }^{23}$ unraveling the effects of substrate on lamellar structure formation and electron transport, ${ }^{24}$ understanding the water intake and swelling in poly(3,4-ethylenedioxythiophene) polystyrene sulfonate (PEDOT:PSS), ${ }^{18}$ just to name a few.

In the present paper, using molecular dynamics simulations, we provide an atomistic understanding of reversible structural phase transitions during electrochemical oxidation and ion injection recently reported by Bischak et al. ${ }^{19}$ in a conjugated polymer with glycolated side chains [2,5-bis(thiophenyl)-1,4bis(2-(2-(2-methoxyethoxy)ethoxy)-ethoxy)benzene] (PB2TTEG), where the polymer switched between two structurally distinct crystalline phases corresponding to different oxidation levels. Our calculations reveal structural changes in the polymer morphology during oxidation and water intake. We demonstrate that the substrate plays an important role in the arrangement of polymer chains, which eventually enables phase transition. This is an important conclusion (often overlooked in the experimental studies) demonstrating that surface engineering can be a crucial factor affecting the device and material functionalities. It is important to stress that the calculated X-ray diffraction curves are not only in qualitative agreement but also in quantitative agreement with the reported experimental data. This outlines the predictive power of computational microscopy of polymer systems that can be utilized for the design of materials and devices with improved performance.

\section{MODEL AND METHODS}

II.I. System under Study. Atomistic molecular dynamics (MD) simulations were carried out to study phase transition in a conducting polymer, poly [2,5-bis(thiophenyl)-1,4-bis(2-(2-(2-methoxyethoxy)ethoxy)ethoxy) benzene] with glycolated side chains known as PB2T-TEG. ${ }^{19}$ One monomer unit of the PB2T-TEG is shown in Figure 1a. The length of one monomer unit of PB2T-TEG in the backbone consisting of two thiophene rings and one benzene ring is $\approx 12 \AA$. Two glycolated side chains of length $\approx 13 \AA$ are attached to the benzene ring. In this way, the maximum width of a single polymer is $\approx 27 \AA$ when the two side chains are fully extended.

The structural phase transition in PB2T-TEG film has been elucidated experimentally by Bischak et al. ${ }^{19}$ using cyclic voltammetry (CV). During the CV experiment, the spin-coated and annealed (at $\sim 430 \mathrm{~K})$ PB2T-TEG film synthesized on $\mathrm{Au}$ substrate was electrochemically oxidized by applying voltage in aqueous electrolyte $(\mathrm{KCl})$. The structural changes in morphology upon oxidation were investigated by X-ray scattering.

We set up our MD simulations to mimic the experimental device fabrication and the CV experiment of Bischak et al. ${ }^{19}$ (see Figure 1b). We first performed simulated annealing (at $\sim 500 \mathrm{~K}$ ) to make the

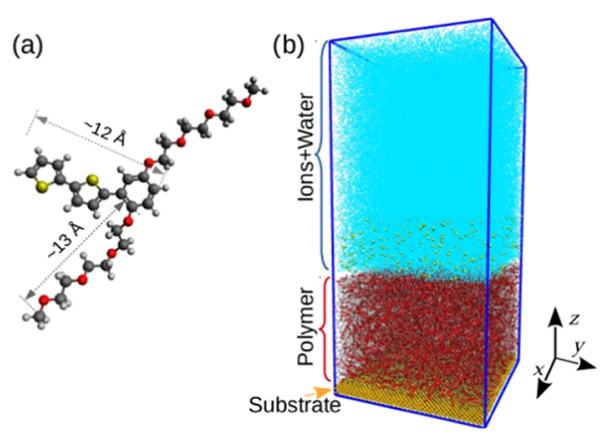

Figure 1. (a) Chemical structure of one monomer unit of PB2T-TEG consisting of two thiophene rings and one benzene ring in the backbone and two glycolated side chains attached with the benzene ring. (b) Initial system of MD simulations consisting a multilayered gold substrate, a dry polymer film, and water with $\mathrm{Cl}^{-}$ions.

PB2T-TEG film, where 250 polymer chains were used to produce a film, with one polymer chain consisting of 10 repeating units. Then, we placed the film in water, letting the film swell. At the same time, we homogeneously oxidized the film by increasing positive charges of the individual polymer chains and placing exact number of $\mathrm{Cl}^{-}$ions outside the film in water. During oxidation, the hydrated $\mathrm{Cl}^{-}$ions enter the polymer film from the electrolyte solution to compensate the excess positive charges in the polymer upon doping. This procedure of a "computational cyclic voltammetry" is the same as those utilized in our previous study to model the ion injection and water intake during electrochemical oxidation in conductive polymers poly-[3,3'-bis(2-(2-(2-methoxyethoxy)ethoxy)ethoxy)-2,2'-bithiophene] $(\mathrm{p}(\mathrm{gT} 2))^{13}$ and poly(3,4-ethylenedioxythiophene) polystyrene sulfonate (PEDOT:PSS). ${ }^{18}$ We performed computational cyclic voltammetry at different oxidation levels $\left(C_{\mathrm{ox}}\right)$ ranging from 0 to $100 \%$. At each oxidation level, partial charges of all of the atoms were calculated using density functional theory (DFT), and the partial charges of all of the charged states are provided in the Supporting Information (see Figure S1).

II.II. Simulation Details. MD simulations were carried out in LAMMPS, ${ }^{25}$ and the initial system was formed using Packmol $^{26}$ and Moltemplate code. ${ }^{27}$ The bonded and nonbonded interactions of the atoms were described using the general AMBER force field (GAFF). ${ }^{28}$ Long-range electrostatic interaction was incorporated via particleparticle particle-mesh solver, ${ }^{29}$ as implemented in LAMMPS. The water molecules were modeled using the transferable intermolecular potential 3P (TIP3P) water model. ${ }^{30}$ During the simulations, the bonds and angles of the water molecules were restrained by the SHAKE algorithm. ${ }^{31}$ Periodic boundary conditions were applied in all three directions. The electrostatic potential (ESP)-derived partial charges $^{32}$ of the atoms of a polymer for all of the charged states (oxidation levels, $C_{\mathrm{ox}}$ ) were calculated using DFT calculations with $\omega \mathrm{B} 97 \mathrm{XD}$ functional ${ }^{33}$ and with $6-31 \mathrm{~g}(\mathrm{~d})$ basis set as implemented in Gaussian. ${ }^{34}$ We calculated the X-ray diffraction (XRD) pattern as implemented in LAMMPS. ${ }^{25,35}$

II.II.I. System without Substrate. The polymer chains were randomly placed in a large simulation box $\left(\approx 80 \times 80 \times 60 \mathrm{~nm}^{3}\right)$ without any position overlap. The simulation box was then deformed to $\approx 14 \times 14 \times 10 \mathrm{~nm}^{3}$, and the system was equilibrated for $5 \mathrm{~ns}$ at $500 \mathrm{~K}$ and $1 \mathrm{~atm}$ in the isothermal-isobaric (NPT) ensemble. In the next step, the system was quenched to room temperature $(300 \mathrm{~K})$ at a cooling rate of $10 \mathrm{~K} / 200 \mathrm{ps}$, and the system was further equilibrated at $300 \mathrm{~K}$ and $1 \mathrm{~atm}$ in the same NPT ensemble. The said process was carried out in Langevin dynamics to incorporate the effect of implicit solvent with a dielectric constant of 5 and a damping coefficient of 3800. To perform the cyclic voltammetry simulations, the selfassembled polymer film was dipped in water (see Figure $2 \mathrm{a}$ ). The film was placed at the center of a simulation box of size $\approx 46 \times 46 \times 36$ $\mathrm{nm}^{3}$, and it was surrounded by 276478 water molecules ( $75 \mathrm{wt} \%$ ). Next, the film was oxidized by gradually increasing the charge of individual polymer chain from 0 to 6 on each chain. At each oxidation 

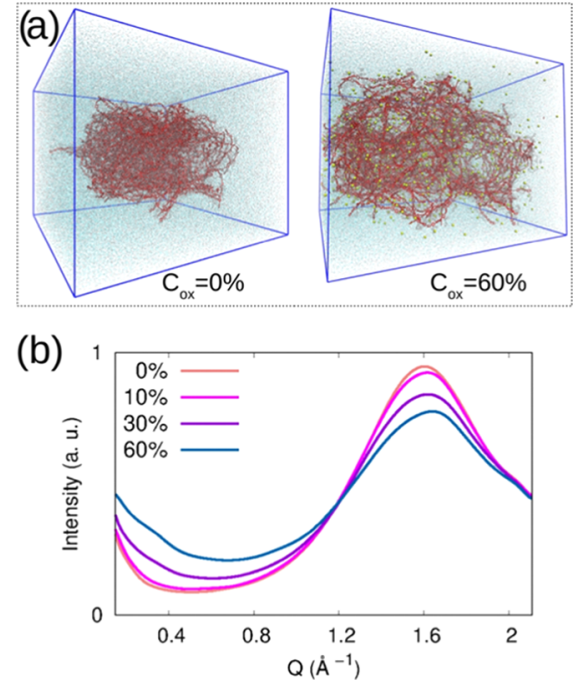

Figure 2. System without substrate. (a) Snapshots of the system for two different oxidation levels, undoped $C_{\mathrm{ox}}=0 \%$ (left) and doped $C_{\mathrm{ox}}$ $=60 \%$ (right). (b) X-ray diffraction pattern calculated from the $\mathrm{MD}$ simulations. The backbones are shown in red, side chains are in gray, water in turquoise, and ions are in gold.

level $\left(C_{\mathrm{ox}}\right)$, the exact number of chloride $\left(\mathrm{Cl}^{-}\right)$ions was added in the water outside the polymer region to make the total system charge zero. The system consisting of the polymer film, water, and ions was equilibrated at $300 \mathrm{~K}$ and $1 \mathrm{~atm}$ in isothermal-isobaric (NPT) ensemble until the total energy reached saturation.
II.II.II. System with Substrate. An $8 \AA$ thick four-layered gold substrate was formed using Visual Molecular Dynamics (VMD) software ${ }^{36}$ with the inorganic builder plugin. The substrate was placed at the bottom of a simulation box, as shown in Figure 1b. A total of 250 polymer chains on top of the gold substrate in a simulation box of $\approx 14 \times 14 \times 10.8 \mathrm{~nm}^{3}$ were first equilibrated in the canonical ensemble (NVT) at $500 \mathrm{~K}$. The whole system was then quenched to room temperature and further equilibrated. To simulate the system with substrate in the NVT ensemble, volume was chosen such that polymer density obtained from the previous NPT simulations (without substrate) is maintained. To perform computational cyclic voltammetry, ions and water were placed above the polymer film, as shown in Figure 1b. Cyclic voltammetry simulations were also carried out in the NVT ensemble. The charge of the individual polymer chains was increased in the same way as discussed previously for the system without substrate.

\section{RESULTS AND DISCUSSION}

First, cyclic voltammetry simulations of the PB2T-TEG polymer were performed without considering the effect of a substrate. The snapshots of the system for the oxidation levels in the $C_{\mathrm{ox}}$ range of $0-60 \%$ are shown in Figure $2 \mathrm{a}$. At $C_{\mathrm{ox}}=$ $0 \%$, there is no water intake by the film from the electrolyte. When the chains become oxidized, the film swells because the hydrated ions move from the electrolyte into the film to compensate the positive charge of the chains (see Figure 2a (right)). The absence of water intake for nonoxidized film and the mechanism of swelling during electrochemical oxidation is similar to a related thiophene-based polymer with glycolated side chains, $\mathrm{p}(\mathrm{gT} 2)$, described in our previous work. ${ }^{13}$ Figure $2 \mathrm{~b}$ shows X-ray diffraction (XRD) curves plotted from the MD
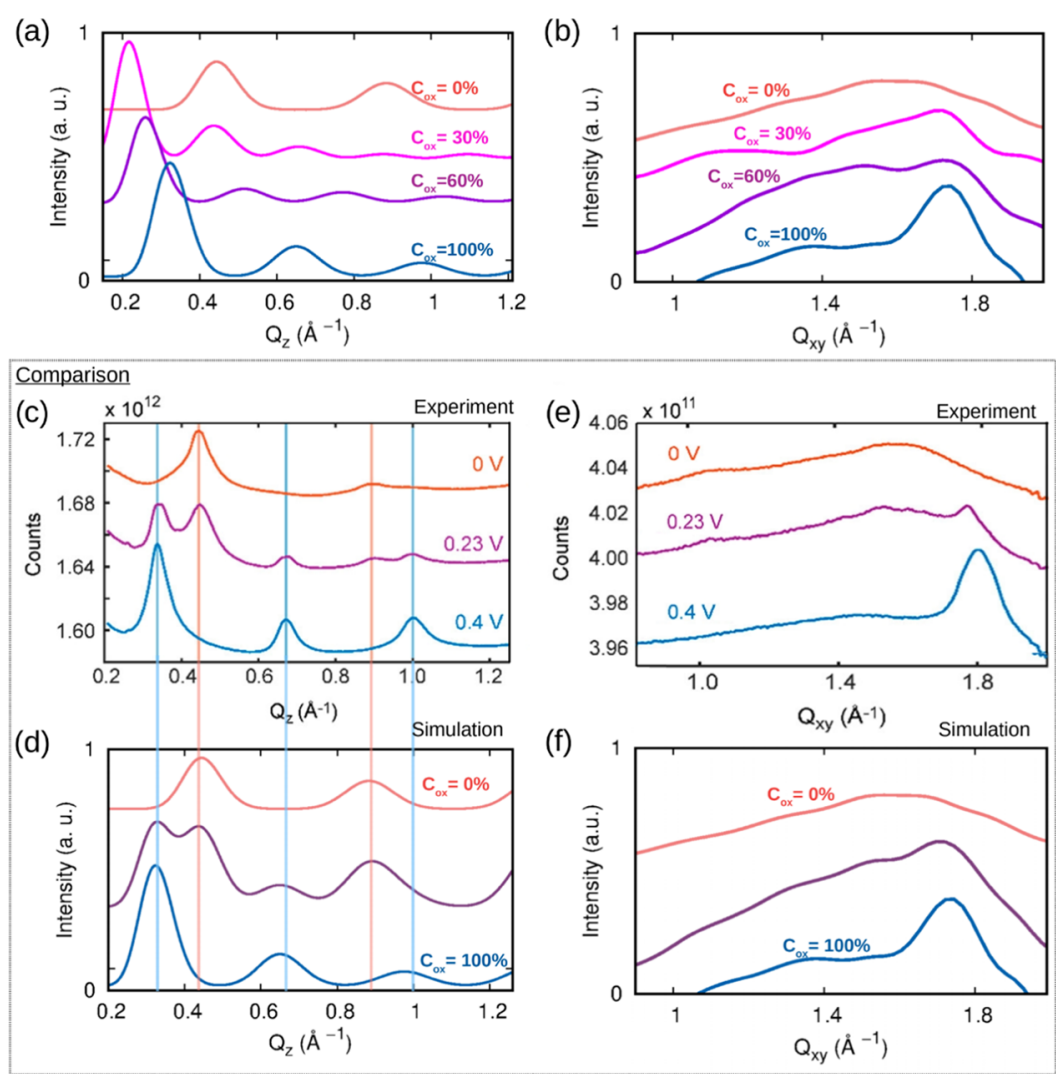

Figure 3. X-ray diffraction (XRD) curve plotted from MD simulations in (a) out-of-plane and (b) in-plane directions. The calculated XRDs are compared with the experiment: ${ }^{19}$ (c, d) Comparison of out-of-plane XRD and (e, f) comparison of in-plane XRD. The images in (c) and (e) are adapted from ref 19 with the permission of the American Chemical Society. 


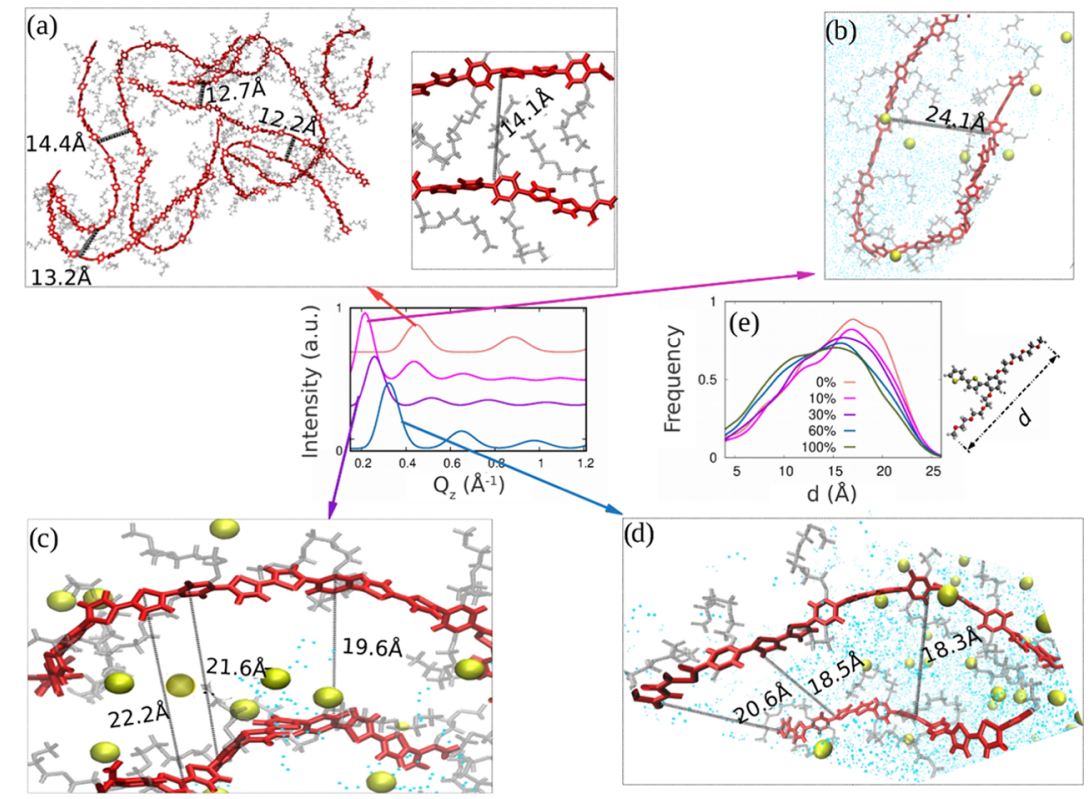

Figure 4. Representative snapshots of the polymer film showing conformational changes upon oxidation and evolution of the lamellar structure. (a) Lamellar stacked chains of a neutral film $\left(C_{o x}=0 \%\right)$; the insets show a closer look at the chains. Ion-intercalated lamellar stacked chains of doped films of (b) $C_{\mathrm{ox}}=30 \%$, (c) $C_{\mathrm{ox}}=60 \%$, and (d) $C_{\mathrm{ox}}=100 \%$. Spacing between lamellar stacking is shown by the dotted lines, and the distance is marked in Ångströms. (e) Distribution of the effective width, $d$, of the polymer for different oxidation levels (left) and schematic definition of $d$ (right). The backbones are shown in red, side chains are in gray, water in turquoise, and ions are in gold. The central inset displaying XRD patterns is the same as in Figure 3a.
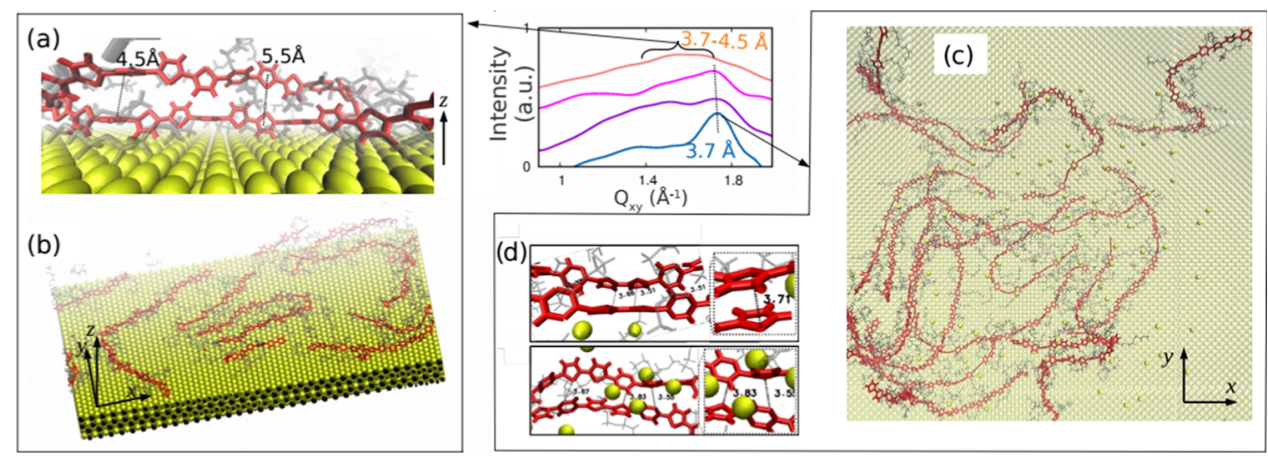

Figure 5. Representative snapshots of the polymer film showing the effect of the substrate on edge-on chain orientation and formation of the $\pi-\pi$ stacking in the in-plane direction. $(a-c)$ Only chains close to the substrate; $(a, b)$ and $(c, d)$ show undoped and fully oxidized $\left(C_{o x}=100 \%\right)$ samples, respectively. (d) Close-up of $\pi-\pi$ stacking chains. The backbones are shown in red, the side chains are in gray, and the ions are in gold; water is not shown. The central inset displaying XRD patterns is the same as in Figure $3 \mathrm{~b}$.

simulations for different oxidation levels. The XRD pattern is rather featureless, exhibiting a broad background peak at $Q \approx$ $1.6 \AA^{-1}$. Although this peak gradually decreases as $C_{\text {ox }}$ increases and the polymer swells, the overall shape of the XRD curve remains practically the same. Hence, the phase transition phenomenon observed in the experiment ${ }^{19}$ (see Figure $3 \mathrm{~d}, \mathrm{f}$ ) is completely missing in the calculated XRD plot. However, it is important to stress that the experimental features and evolution of XRD plots are different between the out-ofplane direction $\left(Q_{z}\right)$ (i.e., the normal to the substrate) and inplane direction $\left(Q_{x y}\right)$. This indicates a strong influence of the substrate, and we therefore conclude that a theoretical understanding of the phase transition requires accounting the effect of the substrate. Note that a crucial role of the substrate in the formation of lamellar stacking and the stacking orientation (face-on vs edge-on) in PEDOT:TOS has been recently outlined via atomistic MD simulation by FrancoGonzalez et al. ${ }^{24}$
In the following, we therefore investigate the electrochemical oxidation of the polymer and the corresponding morphological changes in the system with the substrate. We plot the XRD curve in the out-of-plane, $Q_{z}$ (see Figure $3 a$ ), and in-plane directions, $Q_{x y}$ (see Figure $3 \mathrm{~b}$ ), to elucidate the experimentally observed phase transition for different oxidation levels. Furthermore, we discuss the origin and evolution of the peaks in the XRD patterns by illustrating them with some representative snapshots, as shown in Figures 4 and 5.

Figure $3 \mathrm{a}$ shows the evolution of XRD peaks in the out-ofplane direction $\left(Q_{z}\right)$ as the oxidation level is increased from $C_{\mathrm{ox}}$ $=0$ to $100 \%$. For the undoped (charge-neutral) film, a peak at $Q_{z} \approx 0.45 \AA^{-1}$ is observed. This peak represents the lamellar stacking among the polymer chains with a distance $d_{1}=2 \pi / Q_{z}$ $\approx 14 \AA$, which is close to the length of the side chains $(\approx 13 \AA)$. Figure 4 a shows a closer look of two lamellar stacked chains, where the side-chain interdigitation is clearly envisaged. The intensity of lamellar stacking peak at $Q_{z} \approx 0.45 \AA^{-1}$ 
significantly decreases upon oxidation and completely disappears at higher oxidation levels $C_{\text {ox }}>30 \%$.

At the same time, a new pronounced peak at lower values of $Q_{z}$ (i.e., at a larger lamella stacking distance) appears upon oxidation of the polymer. For $C_{\mathrm{ox}}=30 \%$, a position of this peak is $Q_{z} \approx 0.25 \AA^{-1}\left(d_{1} \approx 25 \AA\right.$, see Figure $\left.3 a\right)$. The increase in the spacing between two lamellar stacked chains (compared to the undoped case) is attributed to the hydrated-ion inclusion. For the oxidized film, the negatively charged ions enter the lamellar stacks to compensate the positive charges of the polymer chains. As a result, the interdigitation of the side chains is eradicated, resulting in an increase in the lamellar stacking from $d_{1} \approx 14 \AA$ at $C_{\mathrm{ox}}=0 \%$ to $d_{1} \approx 25 \AA$ at $C_{\mathrm{ox}}=30 \%$. Note that the distance $d_{1} \approx 25 \AA$ corresponds to approximately a double length of the side chains (see Figure $4 b$ ).

As the oxidation level increases further, the position of this peak shifts to a larger $Q_{z}$ (i.e., the lamella stacking decreases), reaching $Q_{z} \approx 0.35 \AA^{-1}\left(d_{1} \approx 18 \AA\right)$ at $C_{\mathrm{ox}}=100 \%$. Figure $4 \mathrm{~b}-$ $\mathrm{d}$ shows representative snapshots of the polymer morphology illustrating the decrease of the lamella distance as the oxidation level is increased. This happens because at higher doping levels, positively charged polymers attract more counterions (as illustrated in Figure $4 \mathrm{~b}-\mathrm{d}$ ), which increases the overall electrostatic attraction between the chains. Indeed, even though backbones are charged positively, they are effectively attracted to each other because of the presence of negatively charged ions intercalating in the space between them. When the concentration of ions increases, the attractive Coulombic forces between the ions and backbones increase as well. This leads to the enhanced effective attraction between the backbones (mediated by the negative ions between them), which results in a decrease of the lamellar spacing.

To obtain further insight into the conformational changes in the polymer upon oxidation, we calculated the effective width (d) of the polymer, which is defined as a distance between two ends of two side chains attached to the same benzene ring, as illustrated in Figure 4e (right). Figure 4e (left) shows the distributions of $d$ at different oxidation levels. The position of the peak of the distribution is shifted to smaller $d$ as the doping level increases, indicating the decrease of the effective width of the polymer. This is consistent with the decrease of the lamellar stacking of the doped polymer upon oxidation, as discussed above. Note that for an undoped (neutral) film, the side chains in the lamellar stacking are interdigitated. Because of that, despite larger $d$, the lamellar stacking spacing is the smallest for the undoped film.

Let us now discuss the evolution of the in-plane XRD $\left(Q_{x y}\right)$ with the oxidation level. The XRD plot for neutral film shows a rather featureless distribution with a wide peak centered around $Q_{x y} \approx 1.4-1.8 \AA^{-1}$ (corresponding to the distances 4.5-3.7 $\AA$ ) (see Figure $3 \mathrm{~b}$ ). As the oxidation level increases, the peak in $Q_{x y}$ corresponding to the $\pi-\pi$ stacking distance $Q_{x y}$ $\approx 1.75 \AA^{-1}\left(d_{\pi-\pi} \approx 3.6 \AA\right)$ emerges, which becomes more eminent at higher oxidation levels.

The absence of $\pi-\pi$ stacking in undoped films and formation of $\pi-\pi$ stacking at higher oxidation levels are illustrated in the snapshots of the polymer morphology presented in Figure 5. Let us first note that $\pi-\pi$ stacking peaks are present only in the in-plane direction $\left(Q_{x y}\right)$ and are not detected in the out-of-plane direction, $Q_{z}$ (see Figure $3 \mathrm{a}, \mathrm{b})$. This indicates that polymer chains are preferentially arranged edge-on with respect to the surface. ${ }^{37}$ An inspection of the snapshots of the chains situated close to the surface demonstrates that this is indeed the case for all oxidation levels (see Figure $5 a-c)$. The preferential edge-on orientation leads to the formation of a lamellar structure in the out-of-plane direction (Figure 3a). The above observations outline the important role played by the substrate that determines many distinctive features of the film morphology. For further discussion, it is noteworthy to recall that the backbone of PB2T-TEG embraces two different types of $\pi$-conjugated ring, namely, thiophene ring and benzene ring, and therefore, $\pi-\pi$ stacking in the system depends on the orientation of both rings. For the case of the undoped system, polymer chains are attracted to each other due to van der Waals interactions, but they do not form $\pi-\pi$ stacking (see Figure 5a). The thiophene rings are situated on the same plane with spacing $\approx 4.5-5 \AA$ between their centers. In such an arrangement, the overlap between their orbitals is practically absent. The benzene rings form a sandwich-type configuration with spacing $\approx 4.5 \AA$, which is larger than a typical $\pi-\pi$ stacking distance. Besides, they are shifted with respect to each other, and therefore, the overlap of their $\pi$-orbitals is insignificant. Formation of $\pi-\pi$ stacking at a high doping level $\left(C_{\mathrm{ox}}=100 \%\right)$ is illustrated in Figure $5 \mathrm{~d}$. The snapshots demonstrate that both benzene and thiophene rings are arranged in the $\pi-\pi$ stacking. Note that because of the torsion motion of the chains, the rings in the $\pi-\pi$ stacks are not always perfectly parallel to each other.

Let us now compare our calculated results with the experimentally measured XRD patterns. ${ }^{19}$ In the experiment, the film was electrochemically oxidized by applying a voltage from 0 to $+0.7 \mathrm{~V}$, and XRD patterns were measured during the oxidation process. Below $\approx+0.2 \mathrm{~V}$, the film remained undoped and did not show ion uptake. Between +0.2 and $+0.3 \mathrm{~V}$, a rapid increase in dopant concentration (an order of magnitude), lamellar stacking expansion $(\sim 14-18 \AA)$, and a sharp increase in the mass uptake were observed, which altogether indicated that the film has undergone a structural transition from the undoped to doped phase for $V>+0.3 \mathrm{~V}$. Experimentally measured XRD curves from ref 19 are reproduced for convenience in Figure 3c,e. For the undoped film $(V=0)$, the XRD plot shows a lamellar peak at $Q_{z} \approx 0.45 \AA^{-1}\left(d_{1} \approx 14\right.$ $\AA$ ), and for the fully oxidized sample $(V=+0.4 \mathrm{~V})$, the position of the lamellar peak is shifted to $Q_{z} \approx 0.35 \AA^{-1}\left(d_{1} \approx 18 \AA\right)$. It was experimentally shown that in the transition region $(V=$ $+0.23 \mathrm{~V}$ ), the film consists of the phase-separated blend of doped and undoped regions separated by a sharp front. As a result, a corresponding experimental pattern represents a linear superposition of the patterns acquired at 0 and $+0.4 \mathrm{~V}$.

Note that in our calculations, the polymer film was progressively oxidized to different doping levels. Thus, to compare with the experiment, we plot the calculated XRD curves for undoped and fully doped films, and for the transition region, we construct their linear superposition (see Figure $3 \mathrm{~d}, \mathrm{f}$ ). The comparison of the calculated and measured XRD curves (cf. Figure 3d,f and Figure 3c,f) shows that they are not only in qualitative agreement but also in detailed quantitative agreement, for both $Q_{z}$ and $Q_{x y}$. Therefore, we conclude that our simulations can be used to interpret and explain the experimental data. The authors of ref 19 hypothesized that PB2T-TEG undergoes an unzipping/zipping mechanism occurring upon oxidation and reduction. Our simulations presented above unravels the formation of the lamellar structure, and its evolution with the increase of the oxidation level indeed confirms this hypothesis. Note that our simulations show that the lamellar distance changes gradually 
if the oxidation level also changes gradually (see Figure 3a). In the experiment, the oxidation level changes abruptly, causing the observed phase transition. The authors hypothesized that this happens because it can be energetically more favorable to oxidize unzipped regions (i.e., the regions where the interdigitation of the side chains is eradicated due to the ion intake), which, in turn, may lead to nucleation and growth-like kinetics of ion insertion. Based on our calculations, as well as on percolative analysis of the electron transport reported earlier for related conductive polymers, ${ }^{24,38-40}$ we suggest a somehow different perspective, related to the enhancement of the electron mobility in the unzipped regions, as outlined below.

First, we note that a high electron mobility of a polymer represents a prerequisite for the efficient oxidation because holes (polarons) should be able to efficiently travel through the polymer from the electrode to meet the counterions. ${ }^{8}$ In turn, efficient $\pi-\pi$ stackings between the chains represent a prerequisite for high mobility. Indeed, it was demonstrated experimentally and rationalized through multiscale simulations that the high electron mobility can be achieved even in rather amorphous polymers where percolative electron networks through the entire film are formed by polymer chains connected by $\pi-\pi$ stackings. ${ }^{24,38-43}$ As discussed above, $\pi-\pi$ stacking is absent for low oxidation levels (see Figure $3 b$ ), which results in a low electron mobility. Hence, the polymer film remains nonconductive, and therefore its oxidation is difficult. For the higher oxidation levels, the morphology of the system changes and $\pi-\pi$ stacking connection between the chains becomes well established. When this happens, percolative paths become extended through the entire film, which facilitates the oxidation of the chains, which, in turn, leads to the efficient water and ion intake, leading to unzipping of the chains at the polymer-electrolyte interface. Therefore, we conclude that the observed phase transition arises due to an interplay between several factors, including (A) the effect of the substrate leading to the formation of lamellas, (B) unzipping of the interdigitated polymer chains during oxidation and ion intake, and (C) changes in the morphology when $\pi-\pi$ stacking is absent at a low oxidation level and forms at a high oxidation level facilitating the electron mobility and enabling the oxidation of the polymer film.

\section{CONCLUSIONS}

The phase transition in PB2T-TEG polymer thin film exhibiting the conformational changes during electrochemical switching ${ }^{19}$ is theoretically investigated by molecular dynamics simulations mimicking the experimental cyclic voltammetry. To outline the structural changes, we calculate the polymer film morphology and X-ray diffraction (XRD) patterns at different oxidation levels. The calculated XRD patterns not only qualitatively but also quantitatively reproduce the experiment. Our simulations visualize the formation of the lamellar structure and the morphological changes in the film during the phase transition. We conclude that the observed phase transition takes place because of the interplay of several factors, including unzipping of the interdigitated polymer chains during oxidation and the alteration of the morphology of the system in the absence of $\pi-\pi$ stacking between the chains at a low oxidation level and $\pi-\pi$ stacking formation at a high oxidation level. The formation of $\pi-\pi$ stacking leads to an enhancement of the electron mobility, which enables oxidation of the polymer films.
It is noteworthy that the simulation for the same system but without substrate does not show any phase transition or/and structural changes in the system during oxidation and water and ion intake. This demonstrates that the substrate plays an important role in the arrangement of the polymer chains, in particular, in the formation of a lamellar structure, which eventually enables phase transition. This is an important conclusion (often overlooked in the experimental studies) demonstrating that surface engineering can be a crucial factor affecting the device and material functionalities. We finally stress that the excellent quantitative agreement between the calculations and the experiment demonstrates the predictive power of the computational microscopy of the polymer systems, which can be utilized for the design of materials and devices with improved performance.

\section{ASSOCIATED CONTENT}

\section{Supporting Information}

The Supporting Information is available free of charge at https://pubs.acs.org/doi/10.1021/acsaelm.0c00833.

Molecular dynamics simulations and electrostatic potential (ESP)-derived partial charges of the atoms of a polymer for all of the charged states for different oxidation levels (PDF)

\section{AUTHOR INFORMATION}

\section{Corresponding Author}

Igor Zozoulenko - Laboratory of Organic Electronics, ITN, Linköping University, SE-60174 Norrköping, Sweden; (1) orcid.org/0000-0002-6078-3006;

Email: igor.zozoulenko@liu.se

\section{Author}

Sarbani Ghosh - Laboratory of Organic Electronics, ITN, Linköping University, SE-60174 Norrköping, Sweden; (1) orcid.org/0000-0002-3012-910X

Complete contact information is available at:

https://pubs.acs.org/10.1021/acsaelm.0c00833

\section{Notes}

The authors declare no competing financial interest.

\section{ACKNOWLEDGMENTS}

This work was supported by Knut and Alice Wallenberg Foundation, the Swedish Research Council (Project 201704474), and the Swedish Government Strategic Research Area in Materials Science on Advanced Functional Materials at Linköping University (Faculty Grant SFO-Mat-LiU No. 200900971). The computations were performed on resources provided by the Swedish National Infrastructure for Computing (SNIC) at NSC and HPC2N. The authors acknowledge discussion and correspondence with David Ginger and Connor Bischak at earlier stages of this work.

\section{REFERENCES}

(1) Berggren, M.; Crispin, X.; Fabiano, S.; Jonsson, M. P.; Simon, D. T.; Stavrinidou, E.; Tybrandt, K.; Zozoulenko, I. Ion ElectronCoupled Functionality in Materials and Devices Based on Conjugated Polymers. Adv. Mater. 2019, 31, No. e1805813.

(2) Volkov, A. V.; Wijeratne, K.; Mitraka, E.; Ail, U.; Zhao, D.; Tybrandt, K.; Andreasen, J. W.; Berggren, M.; Crispin, X.; Zozoulenko, I. V. Understanding the Capacitance of PEDOT:PSS. Adv. Funct. Mater. 2017, 27, No. 1700329. 
(3) Mortimer, R. J.; Dyer, A. L.; Reynolds, J. R. Electrochromic organic and polymeric materials for display applications. Displays 2006, 27, 2-18.

(4) Xuan, Y.; Sandberg, M.; Berggren, M.; Crispin, X. An allpolymer-air PEDOT battery. Org. Electron. 2012, 13, 632-637.

(5) Rivnay, J.; Inal, S.; Salleo, A.; Owens, R. M.; Berggren, M.; Malliaras, G. G. Organic electrochemical transistors. Nat. Rev. Mater. 2018, 3, No. 17086.

(6) Rivnay, J.; Owens, R. M.; Malliaras, G. G. The Rise of Organic Bioelectronics. Chem. Mater. 2014, 26, 679-685.

(7) Jonsson, A.; Song, Z.; Nilsson, D.; Meyerson, B. A.; Simon, D. T.; Linderoth, B.; Berggren, M. Therapy using implanted organic bioelectronics. Sci. Adv. 2015, 1, No. e1500039.

(8) Berggren, M.; Malliaras, G. G. How conducting polymer electrodes operate. Science 2019, 364, 233-234.

(9) Bießmann, L.; Kreuzer, L. P.; Widmann, T.; Hohn, N.; Moulin, J.-F.; Müller-Buschbaum, P. Monitoring the Swelling Behavior of PEDOT:PSS Electrodes under High Humidity Conditions. ACS Appl. Mater. Interfaces 2018, 10, 9865-9872.

(10) Savva, A.; Wustoni, S.; Inal, S. Ionic-to-electronic coupling efficiency in PEDOT:PSS films operated in aqueous electrolytes. J. Mater. Chem. C 2018, 6, 12023-12030.

(11) Kim, B.; Na, J.; Lim, H.; Kim, Y.; Kim, J.; Kim, E. Robust High Thermoelectric Harvesting Under a Self-Humidifying Bilayer of Metal Organic Framework and Hydrogel Layer. Adv. Funct. Mater. 2019, 29, No. 1807549.

(12) Sarkar, B.; Jaiswal, M.; Satapathy, D. K. Swelling kinetics and electrical charge transport in PEDOT:PSS thin films exposed to water vapor. J. Phys.: Condens. Matter 2018, 30, No. 225101.

(13) Gladisch, J.; Stavrinidou, E.; Ghosh, S.; Giovannitti, A.; Moser, M.; Zozoulenko, I.; McCulloch, I.; Berggren, M. Reversible Electronic Solid-Gel Switching of a Conjugated Polymer. Adv. Sci. 2020, 7, No. 1901144.

(14) Zhanshayeva, L.; Favaron, V.; Lubineau, G. Macroscopic Modeling of Water Uptake Behavior of PEDOT:PSS Films. ACS Omega 2019, 4, 21883-21890.

(15) Cendra, C.; Giovannitti, A.; Savva, A.; Venkatraman, V.; McCulloch, I.; Salleo, A.; Inal, S.; Rivnay, J. Role of the Anion on the Transport and Structure of Organic Mixed Conductors. Adv. Funct. Mater. 2019, 29, No. 1807034.

(16) Wieland, M.; Dingler, C.; Merkle, R.; Maier, J.; Ludwigs, S. Humidity-Controlled Water Uptake and Conductivities in Ion and Electron Mixed Conducting Polythiophene Films. ACS Appl. Mater. Interfaces 2020, 12, 6742-6751.

(17) Savva, A.; Hallani, R.; Cendra, C.; Surgailis, J.; Hidalgo, T. C.; Wustoni, S.; Sheelamanthula, R.; Chen, X.; Kirkus, M.; Giovannitti, A.; Salleo, A.; McCulloch, I.; Inal, S. Balancing Ionic and Electronic Conduction for High-Performance Organic Electrochemical Transistors. Adv. Funct. Mater. 2020, 30, No. 1907657.

(18) Modarresi, M.; Mehandzhiyski, A.; Fahlman, M.; Tybrandt, K.; Zozoulenko, I. Microscopic Understanding of the Granular Structure and the Swelling of PEDOT:PSS. Macromolecules 2020, 53, 62676278 .

(19) Bischak, C. G.; Flagg, L. Q.; Yan, K.; Rehman, T.; Davies, D. W.; Quezada, R. J.; Onorato, J. W.; Luscombe, C. K.; Diao, Y.; Li, C.Z.; Ginger, D. S. A Reversible Structural Phase Transition by Electrochemically-Driven Ion Injection into a Conjugated Polymer. J. Am. Chem. Soc. 2020, 142, 7434-7442.

(20) Lu, B.; Yuk, H.; Lin, S.; Jian, N.; Qu, K.; Xu, J.; Zhao, X. Pure PEDOT:PSS hydrogels. Nat. Commun. 2019, 10, No. 1043.

(21) Moser, M.; Hidalgo, T. C.; Surgailis, J.; Gladisch, J.; Ghosh, S.; Sheelamanthula, R.; Thiburce, Q.; Giovannitti, A.; Salleo, A.; Gasparini, N.; Wadsworth, A.; Zozoulenko, I.; Berggren, M.; Stavrinidou, E.; Inal, S.; McCulloch, I. Side Chain Redistribution as a Strategy to Boost Organic Electrochemical Transistor Performance and Stability. Adv. Mater. 2020, 32, No. 2002748.

(22) Lee, E. H.; Hsin, J.; Sotomayor, M.; Comellas, G.; Schulten, K. Discovery Through the Computational Microscope. Structure 2009, 17, 1295-1306.
(23) Alessandri, R.; Uusitalo, J. J.; de Vries, A. H.; Havenith, R. W. A.; Marrink, S. J. Bulk Heterojunction Morphologies with Atomistic Resolution from Coarse-Grain Solvent Evaporation Simulations. J. Am. Chem. Soc. 2017, 139, 3697-3705.

(24) Franco-Gonzalez, J. F.; Rolland, N.; Zozoulenko, I. V. Substrate-Dependent Morphology and Its Effect on Electrical Mobility of Doped Poly(3,4-ethylenedioxythiophene) (PEDOT) Thin Films. ACS Appl. Mater. Interfaces 2018, 10, 29115-29126.

(25) Plimpton, S. Fast Parallel Algorithms for Short-Range Molecular Dynamics. J. Comput. Phys. 1995, 117, 1-19.

(26) Martínez, L.; Andrade, R.; Birgin, E. G.; Martínez, J. M. PACKMOL: A package for building initial configurations for molecular dynamics simulations. J. Comput. Chem. 2009, 30, 21572164.

(27) Jewett, A.; Lambert, J. Moltemplate (Code), http://www. moltemplate.org/ (downloaded: January 2018).

(28) Wang, J.; Wolf, R. M.; Caldwell, J. W.; Kollman, P. A.; Case, D. A. Development and testing of a general amber force field. J. Comput. Chem. 2004, 25, 1157-1174.

(29) Hockney, R. W.; Eastwood, J. W. Computer Simulations Using Particles; Taylor \& Francis: New York, NY, 1988.

(30) Jorgensen, W. L.; Chandrasekhar, J.; Madura, J. D.; Impey, R. W.; Klein, M. L. Comparison of simple potential functions for simulating liquid water. J. Chem. Phys. 1983, 79, 926-935.

(31) Ryckaert, J.-P.; Ciccotti, G.; Berendsen, H. J. C. Numerical integration of the cartesian equations of motion of a system with constraints: molecular dynamics of n-alkanes. J. Comput. Phys. 1977, 23, 327-341.

(32) Singh, U. C.; Kollman, P. A. An approach to computing electrostatic charges for molecules. J. Comput. Chem. 1984, 5, 129145.

(33) Lin, Y.-S.; Li, G.-D.; Mao, S.-P.; Chai, J.-D. Long-Range Corrected Hybrid Density Functionals with Improved Dispersion Corrections. J. Chem. Theory Comput. 2013, 9, 263-272.

(34) Frisch, M. J.; Trucks, G. W.; Schlegel, H. B.; Scuseria, G. E.; Robb, M. A.; Cheeseman, J. R.; Scalmani, G.; Barone, V.; Petersson, G. A.; Nakatsuji, H.; Li, X.; Caricato, M.; Marenich, A. V.; Bloino, J.; Janesko, B. G.; Gomperts, R.; Mennucci, B.; Hratchian, H. P.; Ortiz, J. V.; Izmaylov, A. F.; Sonnenberg, J. L.; Williams; Ding, F.; Lipparini, F.; Egidi, F.; Goings, J.; Peng, B.; Petrone, A.; Henderson, T.; Ranasinghe, D.; Zakrzewski, V. G.; Gao, J.; Rega, N.; Zheng, G.; Liang, W.; Hada, M.; Ehara, M.; Toyota, K.; Fukuda, R.; Hasegawa, J.; Ishida, M.; Nakajima, T.; Honda, Y.; Kitao, O.; Nakai, H.; Vreven, T.; Throssell, K.; Montgomery, J. A., Jr.; Peralta, J. E.; Ogliaro, F.; Bearpark, M. J.; Heyd, J. J.; Brothers, E. N.; Kudin, K. N.; Staroverov, V. N.; Keith, T. A.; Kobayashi, R.; Normand, J.; Raghavachari, K.; Rendell, A. P.; Burant, J. C.; Iyengar, S. S.; Tomasi, J.; Cossi, M.; Millam, J. M.; Klene, M.; Adamo, C.; Cammi, R.; Ochterski, J. W.; Martin, R. L.; Morokuma, K.; Farkas, O.; Foresman, J. B.; Fox, D. J. Gaussian 16, revision B.01; Gaussian, Inc.: Wallingford, CT, 2016.

(35) Coleman, S. P.; Spearot, D. E.; Capolungo, L. Virtual diffraction analysis of $\mathrm{Ni}\left[\begin{array}{lll}0 & 1 & 0\end{array}\right]$ symmetric tilt grain boundaries. Modell. Simul. Mater. Sci. Eng. 2013, 21, No. 055020.

(36) Humphrey, W.; Dalke, A.; Schulten, K. VMD: Visual molecular dynamics. J. Mol. Graphics 1996, 14, 33-38.

(37) Müller-Buschbaum, P. The Active Layer Morphology of Organic Solar Cells Probed with Grazing Incidence Scattering Techniques. Adv. Mater. 2014, 26, 7692-7709.

(38) Rolland, N.; Franco-Gonzalez, J. F.; Volpi, R.; Linares, M.; Zozoulenko, I. V. Understanding morphology-mobility dependence in PEDOT:TOS. Phys. Rev. Mater. 2018, 2, No. 045605.

(39) Rolland, N.; Franco-Gonzalez, J. F.; Zozoulenko, I. Can Mobility Negative Temperature Coefficient Be Reconciled with the Hopping Character of Transport in Conducting Polymers? ACS Appl. Polym. Mater. 2019, 1, 2833-2839.

(40) Rolland, N.; Modarresi, M.; Franco-Gonzalez, J. F.; Zozoulenko, I. Large scale mobility calculations in PEDOT (Poly(3,4-ethylenedioxythiophene)): Backmapping the coarse- 
grained MARTINI morphology. Comput. Mater. Sci. 2020, 179, No. 109678.

(41) Noriega, R.; Rivnay, J.; Vandewal, K.; Koch, F. P. V.; Stingelin, N.; Smith, P.; Toney, M. F.; Salleo, A. A general relationship between disorder, aggregation and charge transport in conjugated polymers. Nat. Mater. 2013, 12, 1038.

(42) Zhang, X.; Bronstein, H.; Kronemeijer, A. J.; Smith, J.; Kim, Y.; Kline, R. J.; Richter, L. J.; Anthopoulos, T. D.; Sirringhaus, H.; Song, K.; Heeney, M.; Zhang, W.; McCulloch, I.; DeLongchamp, D. M. Molecular origin of high field-effect mobility in an indacenodithiophene-benzothiadiazole copolymer. Nat. Commun. 2013, 4, No. 2238.

(43) Wang, S.; Fabiano, S.; Himmelberger, S.; Puzinas, S.; Crispin, X.; Salleo, A.; Berggren, M. Experimental evidence that short-range intermolecular aggregation is sufficient for efficient charge transport in conjugated polymers. Proc. Natl. Acad. Sci. U.S.A. 2015, 112, 1059910604. 